\section{Competencias interculturales en la atención primaria de salud: un desafío para la educación superior frente a contextos de diversidad cultural}

\author{
Intercultural skills in primary health care: a \\ challenge for higher education in contexts of \\ cultural diversity
}

\section{Competências interculturais na atenção primaria à saúde: um desafio para a educação superior frente a contextos de diversidade cultural}

Lizet Veliz-Rojas 1

Andrés Felipe Bianchetti-Saavedra 2

Marta Silva-Fernández 3

doi: 10.1590/0102-311X00120818

\section{Resumen}

En la sociedad actual están presentes cuatro fenómenos articulados que determinan la necesidad de desarrollar competencias interculturales en el ámbito de la atención primaria de salud: los movimientos migratorios, las diferentes etnias, la diversidad cultural y la construcción social y cultural del sistema médico occidental. La realidad multicultural de los contextos en que se ejerce la atención sanitaria exige incorporar modelos teóricos integradores que permitan comprenderla para brindar una atención en salud oportuna, de calidad $y$ pertinente a las comunidades a quienes dirige sus acciones, considerando la diversidad existente. En este sentido, resulta necesario el despliegue y la enseñanza de competencias interculturales en los equipos y estudiantes del área de salud, que permitan sustentar la atención y los cuidados desde una perspectiva holística. La intervención educativa en competencia intercultural debe realizarse desde un enfoque global, integrador y transdisciplinario, que permita intervenir desde las actitudes, los sentimientos, las conductas sociales y las pautas culturales a nivel individual, institucional y social. El presente ensayo tiene como propósito reflexionar acerca de la importancia del aprendizaje $y$ desarrollo de dichas competencias en los equipos de salud de atención primaria y enumerar un listado de estrategias con las que es posible adquirirlas en los procesos formativos previos, entendiendo que las competencias son producto de las experiencias y se pueden transformar como resultado de un proceso.

Competencia Cultural; Diversidad Cultural; Atención Primaria de Salud; Educación Superior; Estudiantes del Área de la Salud

\section{Correspondencia}

A. F. Bianchetti-Saavedra

Universidad San Sebastián

Lientur 1457, Concepción / Concepción - 4080871, Chile.

abianchettis@docente.uss.cl

1 Universidad de La Serena, La Serena, Chile.

2 Universidad San Sebastián, Concepción, Chile.

3 Universidad Austral de Chile, Valdivia, Chile. 


\section{Introducción}

En la dimensión del proceso salud-enfermedad, se encuentra una diversidad de conocimientos, prácticas e instituciones para promover la salud, prevenir, curar y rehabilitar a las personas enfermas. Esta diversidad se constituye en campos simbólicamente mediados que las personas reproducen, construyen y transforman a través de relaciones de poder situadas en un contexto social determinado ${ }^{1}$. Sin embargo, en la cotidianidad de los equipos de salud de la atención primaria, es común entender los conocimientos, las prácticas y los valores culturales como una realidad propia de las comunidades y, de manera negativa, como factores de riesgo que se deben erradicar o cambiar. Esta mirada excluyente no considera que el contexto sociocultural está mediado a la experiencia humana 1.

La Declaración de Alma-Ata (1978) reconoce la salud como un derecho humano fundamental, considerando inaceptable las desigualdades e inequidades respecto a la salud de las poblaciones. Admite el papel de los Estados en el cuidado de la salud, como el de las comunidades como actores de derechos, y del deber en participar en las acciones y políticas de salud. En esta misma línea, propone a la atención primaria en salud como estrategia central para mejorar la salud y bienestar de la población, al entenderla como asistencia sanitaria esencial, basada en métodos y prácticas científicamente fundamentadas y socioculturalmente aceptadas al alcance de todas las personas y comunidades, mediante su plena participación y a un costo que los Estados puedan soportar a través de la autorresponsabilidad y autodeterminación 1,2. En este sentido, se comprende, por un lado, la atención primaria de salud como una consecuencia del contexto sociocultural, político y económico donde está inserta, y por otro lado, el reconocimiento de la producción cultural de los sistemas de salud y comunidades.

La entrega de prestaciones y cuidados en salud en el ámbito de la diversidad cultural debe incluir la dimensión cultural en el contexto biomédico, para ello es necesario posicionarse teóricamente, en relación a los conceptos de cultura y relativismo cultural, puesto que ambos entregan una mirada descolonizadora respecto al modelo biomédico hegemónico 3. Así, Langdon \& Wiik 4 (p. 462) definen el concepto de cultura como "un conjunto de elementos que median y califican cualquier actividad física o mental que no sea determinada por la biología y que sea compartida por diferentes miembros de un grupo social", destacando como elementos constitutivos los valores, símbolos, normas y prácticas. El relativismo cultural, desde una perspectiva antropológica, entiende que las prácticas socialmente aceptadas (o la moralidad de dichas prácticas) difieren de cultura a cultura; en otras palabras, la comprensión de culturas enfatizando la diferencia no es necesariamente crítica 5. Es lo opuesto al etnocentrismo, es decir, la tendencia a evaluar e interpretar las creencias y costumbres de otras sociedades desde la propia cultura. El relativismo cultural, entonces, permite concebir holística y comparativamente una cultura, por tanto, la diversidad cultural adquiere una auténtica dimensión, en tanto permite, como lo señala Geertz 6, comprender "al otro" desde el contexto de su propia cultura, partiendo del supuesto que todas las culturas son iguales y ninguna es superior a otra.

En este aspecto, Leininger \& McFarland 7 (p. 27), en su teoría de la diversidad y de la universalidad de los cuidados culturales, establecen que el conocimiento y el análisis de las diferentes culturas del mundo, desde una perspectiva inter y transcultural, tiene como objetivo "mejorar y proporcionar cuidados en salud culturalmente congruentes a las personas, que sean beneficiosos y útiles para la persona, la familia o comunidad", esto en función de las concepciones y valoraciones asociadas a los cuidados, la expresión y las creencias sobre salud y la enfermedad, y el modelo de conductas. Por un lado, la perspectiva intercultural señala el carácter existencial de los lazos sociales que necesitan ser re-conceptualizados, debido a la diversidad étnica y cultural de la sociedad actual 8. Por otro lado, la trasculturalidad es el punto donde la interacción entre diferentes culturas puede trascender no sólo sus fronteras, sino también sus intersticios, y dar lugar a un nuevo espacio cultural en común 8 .

Desde el pensamiento biomédico, en los sistemas sanitarios occidentales, la cultura es visualizada como un factor de riesgo, un elemento negativo, o la razón por la cual los pacientes o las comunidades no son adherentes, es decir, no siguen las indicaciones o ciertas prácticas sanitarias. En este sentido, los servicios y equipos de salud adquieren un papel hegemónico que representa lo verdadero y lo correcto, y las prácticas y cosmovisiones diferentes representan lo falso o riesgoso 1,4.

En este sentido, surge la importancia de que los equipos de salud de la atención primaria incorporen transversalmente en sus prácticas la dimensión cultural en el trabajo con las personas y comunidades que atienden. El desarrollo de esta sensibilidad cultural se traduce imponderablemente a la 
enseñanza y aprendizaje de competencias interculturales, las cuales son entendidas como comportamientos y comunicación apropiada y efectiva en situaciones interculturales 9 .

$\mathrm{Al}$ respecto, el presente ensayo tiene como propósito reflexionar acerca de la importancia del aprendizaje y desarrollo de dichas competencias en los equipos de salud de atención primaria, y enumerar un listado de estrategias con las que es posible adquirirlas en los procesos formativos previos, entendiendo que las competencias son producto de las experiencias y se pueden transformar como resultado de un proceso.

\section{Desarrollo}

\section{La importancia del aprendizaje de competencias interculturales en salud}

En la sociedad latinoamericana actual, están presentes cuatro fenómenos articulados que determinan la necesidad de enseñar y desplegar competencias interculturales en el ámbito de la atención primaria de salud: (i) las migraciones; (ii) las distintas etnias; (iii) la diversidad cultural; y (iv) el modelo biomédico inserto en el sistema de salud occidental.

El primer fenómeno, los movimientos migratorios, constituye un desafío global de importantes consecuencias para la sociedad. La principal causa de la migración es la económica, por lo que gran parte de la población inmigrante procede de contextos muy diversos culturalmente y de situaciones socioeconómicas más desfavorecidas. Este fenómeno desencadena una alta presión en los servicios de salud de primer nivel, ya que las migraciones se superponen con la desigualdad y la exclusión social, como factores determinantes, con los que deben lidiar las prestaciones sanitarias ofrecidas en la atención primaria ante los escasos recursos que existen en este nivel de atención 10,11.

En el caso de Chile, una proporción significativa de esta comunidad migrante se encuentra en un contexto de vulnerabilidad y segregación social, dada principalmente por su alta concentración en comunas periféricas y de bajo ingreso, lo que se traduce en un restringido acceso a servicios públicos de calidad. La alta exposición a la inseguridad ciudadana y la inserción laboral está caracterizada por la segmentación y la precariedad. Se adiciona a esto la discriminación y el racismo, que si bien no es explícito, se manifiesta principalmente hacia el color de la piel, señalada por los propios haitianos migrantes como la principal barrera en las interacciones sociales con los chilenos 12 .

En general, la experiencia migratoria es muy compleja y exigente, pudiendo vulnerar los derechos humanos, el bienestar y la salud de las personas. Se ha señalado que la condición migratoria, inserta en el contexto de vulnerabilidad social, tiene una relación directa con las desigualdades en salud en el interior de un país. Los principales factores que influyen en la salud de las personas migrantes son: los procesos de migración; asimilación y aculturación; condiciones sociales y laborales; acceso y calidad en la atención en servicios de salud; idioma; costumbres, prácticas y creencias en salud; procesos sociales de inclusión y exclusión; estigma y discriminación 13 .

Acorde a lo anterior, en los establecimientos de salud chilenos, se han reportado algunas situaciones que dificultan a los migrantes obtener un mejor bienestar en salud, tales como: trato discriminatorio y racismo, lo que muchas veces desincentiva la concurrencia de los extranjeros a los centros de salud; el desconocimiento de los migrantes sobre sus derechos y deberes y del funcionamiento del sistema de salud; la falta de protocolos o reglamentación sobre los derechos asegurados, traducidos en algunos casos en interpretaciones restrictivas que vulneran los derechos de los migrantes; y por último, la denegación de acceso a los servicios de salud a migrantes en situación irregular por no contar con su cédula de identidad vigente 14,15 .

Es justamente en estos contextos migratorios cuando las competencias interculturales adquieren relevancia, tal como lo ha evidenciado el estudio realizado por Bernales et al. 13, respecto a las prácticas de los funcionarios de centros de salud públicos. Se entrevistó a 101 funcionarios en ocho comunas de Chile que concentran la mayor densidad de migrantes con altos índices de vulnerabilidad. Uno de los principales resultados dio cuenta de la carencia de competencias interculturales por parte de los funcionarios, lo que se tradujo en una atención en salud carente de prácticas culturalmente pertinentes a las necesidades de la comunidad migrante. Los funcionarios se enfrentan a un mundo culturalmente ajeno y desconocido, el cual han ido abordando a partir de su creatividad y flexibilidad 
aplicadas a sus experiencias laborales. Esto evidencia claramente la necesidad de recibir formación específica, respecto al desarrollo de estrategias en salud que sean pertinentes a la diferencia cultural con diversas comunidades. En otras palabras, los funcionarios necesitan ser formados en el despliegue de competencias interculturales que les permitan comprender la cultura, costumbres, prácticas y creencias de la persona migrante que se está atendiendo.

El segundo fenómeno se refiere a las concepciones que diferentes etnias tienen sobre el proceso salud-enfermedad. Las diferentes etnias presentes en las comunidades visualizan el proceso saludenfermedad holísticamente, lo que ha permitido implementar diversas formas de sanación, las cuales no son reconocidas habitualmente por los sistemas sanitarios 16,17. Las diferencias étnicas se asocian a una fuerte impronta racista 10 , pobreza económica y marginalidad social 18 . Acorde a lo anterior, la Organización Panamericana de la Salud (OPS) 19, en el año 1996, firmó un acuerdo con el Parlamento Indígena de las Américas, en donde se estableció la preparación de una agenda legislativa para las políticas en salud y la producción de leyes que protegiesen las tradiciones indígenas. Al respecto, diversos países latinoamericanos se plantearon la necesidad de desarrollar políticas públicas en salud, con el propósito de avanzar hacia la construcción colectiva de un modelo de salud con enfoque intercultural, y a una mayor participación de los pueblos indígenas en los programas de salud 10,18,20.

Las políticas públicas, y por ende, los programas de salud deberían ser construidos incorporando la dimensión intercultural de manera transversal. Una propuesta conceptual a ser incorporada tiene relación con el buen vivir asociado al desarrollo local, el cual da cuenta de la idea de una vida que no es ni mejor ni peor que la de otros, ni un continuo desvivir por mejorarla, sino simplemente buena en términos definidos por la propia cultura. Este elemento incorpora el elemento comunitario, por lo que se podría traducir como "buen convivir". Esto significa que una sociedad orientada al buen vivir para todos resulta en armonía interna con respecto a los sistemas ecológicos/medioambientales. Una importante consecuencia del buen vivir es el desarrollo local, fomentando a su vez el respeto interétnico entre los pueblos que comparten un contexto geográfico 20.

El buen vivir, traducción desde el quechua de Sumak Kawsay, se contrapone al significado de calidad de vida y bienestar propuesto por el modelo capitalista. El Sumak Kawsay se caracteriza por: la valoración de las relaciones comunitarias en el uso de los bienes; la producción de un trabajo colectivo/comunitario; la promoción de la ritualización y redistribución del excedente, renunciando a la acumulación; y por una reciprocidad sacralizada con la naturaleza, manifestada por la armonía entre ser humano y naturaleza 21 . En este sentido, surge la necesidad que el equipo de salud establezca estrategias que apunten a contribuir al buen vivir desde una cosmovisión holística 22.

A pesar del desarrollo de programas y políticas públicas interculturales en salud, que apuntan a mejorar las condiciones en salud de los pueblos originarios, diversos autores han señalado distintas dificultades en la aplicación de estas, entre las que se encuentran: la ausencia de referentes teóricos, respecto al significado de interculturalidad en salud; las implicancias prácticas que tiene el desarrollo de este enfoque en los equipos de salud, debido especialmente al no reconocimiento de la medicina tradicional y de los estilos de vida de las diversas etnias; el escaso conocimiento de las condiciones estratégicas de las comunidades indígenas para implementar las políticas públicas de salud intercultural 18; y la escaza participación que tienen los grupos étnicos en el diseño y organización de los programas y actividades en salud 23 .

Acorde a lo anterior, emerge la necesidad de que la mirada hegemónica del cuidado de la salud transite hacia la valoración de los distintos sistemas de salud y cuidados establecidos por los distintos pueblos originarios. Desde la formación de pregrado de los profesionales de la salud se deberían disenar e implementar programas y estrategias pedagógicas transversales que den cuenta de las distintas cosmovisiones en el cuidado de la salud 24.

El tercer fenómeno, es la diversidad cultural que se encuentra en todas las comunidades en las cuales los equipos de salud de atención primaria deben ejercer los cuidados. La diversidad cultural "comprende el conjunto de rasgos distintivos, espirituales, materiales, intelectuales y afectivos que caracterizan a una sociedad o grupo social, y abarca los modos de vida, las formas de vivir juntos, los sistemas de valores, las tradiciones y creencias" 25 (p. 18). El nuevo panorama de multiculturalidad, propia de los países de América Latina, está dado por la diversidad cultural en el interior de cada país y de cada comunidad en términos de diferencias entre: zonas rurales, urbanas, niveles sociales, económicos, étnicos, religiosos, entre otros, lo que puede generar confusión o choque para muchos profesionales de la salud. Por un lado, les 
resulta difícil comprender las creencias y prácticas en salud de las personas que atienden y, por otro, les preocupa el poco éxito en la adherencia a los tratamientos 26 . Cada persona tiene una percepción $a$ priori de la diversidad cultural, que se puede mirar como una oportunidad o como amenaza. Estas percepciones individuales y colectivas están presentes y tienen influencia en la forma como se relacionan los equipos de salud, y las personas atendidas en los servicios de salud, que pueden manifestarse en desconfianza, rechazo, miedo, o por el contrario pueden generar intercambio, complicidades, apoyo, enriquecimiento y construcciones comunes 27 . En este sentido, se hace necesario que los servicios de la atención primaria en salud trabajen gestionando la diversidad cultural, promoviendo la inclusión en el medio social en donde actúan, integrando a la comunidad en un contexto de coexistencia entre la individualidad y universalidad.

Otro elemento a considerar es la construcción social y cultural del sistema médico occidental que se ha plasmado como modelo biomédico ${ }^{28}$. La mirada biomédica se ha establecido mundialmente como un modelo sanitario capaz de resolver la mayoría de los problemas de salud de la población, independientemente de los contextos sociales y culturales en que se desarrolle la enfermedad 18. Esto resulta en un proceso de educación de los profesionales de la salud, como un espacio de reproducción de jerarquías, rutinas institucionales, modos de pensar y comunicar, desde una perspectiva hegemónica hacia el cuerpo humano, sus funciones y dolencias ${ }^{10}$. En el modelo biomédico, se tiende a reproducir y amplificar mecanismos jerárquicos que establecen relaciones autoritarias que minimizan la cultura, creencias, tradiciones y costumbres de la comunidad, respecto a la cultura de los prestadores sanitarios 10 . Algunos proyectos e investigaciones en salud dan cuenta de las dificultades a las que se enfrentan los equipos de salud, puesto que no consideran la cultura de los usuarios en el proceso de atención. Situaciones como el rechazo y la baja adherencia al tratamiento, conflictos de poder entre los agentes terapéuticos tradicionales y comunitarios con el equipo sanitario, maltrato y discriminación de los usuarios, entre otros, se presentan en el quehacer diario del ámbito sanitario público 10,18. En este escenario, la construcción de cambios orientados a desarrollar y fortalecer las competencias interculturales del equipo de salud, y la búsqueda de estrategias que articulen e integren los distintos enfoques y cuidados de la salud, permitirá avanzar hacia un sistema sanitario más permeable y flexible a la realidad cultural de las personas, familia y comunidad.

\section{Desarrollo de competencias interculturales en la atención primaria de salud para favorecer el bienestar y alcanzar el buen vivir}

Este contexto de globalización, donde están presentes las migraciones, la diversidad cultural, las diferentes etnias y la cultura organizacional de los sistemas médicos occidentales, supone que las estrategias y acciones en salud no pueden quedar sujetas a una visión simplista y estática de la sociedad. Lo anterior determina no sólo reconocer la complejidad de este contexto multicultural, sino también lo que demanda para fomentar acciones que permitan la participación e interacción armónica entre las comunidades y los equipos de salud, a partir del significado y comprensión de la diversidad.

En este sentido, la interculturalidad se plantea como un modelo integral para atender la diversidad cultural que se concibe como una estrategia sociopolítica propositiva, expresada en acciones tendientes al reconocimiento de las posibilidades y riquezas de la diversidad, el sostenimiento de las particularidades y la lucha contra las desigualdades instaladas en la sociedad, con el propósito de aportar en la solución de conflictos entre culturas y a la transformación de un marco estructural que origina inequidad política, socioeconómica y cultural 8. La interculturalidad conlleva una posición epistemológica que considera lo diverso como un aspecto positivo, como fuente de desarrollo personal y social, al apostar por una construcción de sociedades más equitativas y participativas, por lo tanto, la perspectiva de interculturalidad en salud no puede ser reducida a la atención de las necesidades de grupos que sean objeto de exclusión social, sino que apunta a una transformación que busca el tránsito de una dinámica social, movida por la dominación y negación de las diferencias culturales, a una dinámica que promueva estrategias en salud que faciliten la afirmación identitaria, el acercamiento y la interacción efectiva entre las comunidades y los equipos de salud en equidad de condiciones 29.

La realidad multicultural de los contextos en que se ejerce la atención sanitaria en la atención primaria exige incorporar, en el cuerpo de conocimientos que la sustentan, modelos integradores que permitan comprenderla para brindar una atención en salud oportuna y pertinente a las comunidades 
a quienes dirige sus acciones, considerando la diversidad existente. En este sentido, resulta interesante el desarrollo de competencias interculturales en los equipos de salud de atención primaria, que permitan sustentar la praxis en salud desde una perspectiva integradora y holística para el alcance de un mayor bienestar en salud y el buen vivir.

En el caso de Chile, la atención primaria de salud se basa en el modelo integral de salud familiar y comunitario, que se sustenta en tres principios irrenunciables: (i) centralidad en las personas, considerando la corresponsabilidad del cuidado entre el equipo de salud, las personas y comunidad; (ii) integralidad en la atención, que articula la promoción, la prevención y la rehabilitación desde un abordaje integral de las personas y comunidad; y (iii) continuidad del cuidado, que se relaciona con la conexión y seguimiento del cuidado en todas las etapas del ciclo vital y contexto de la atención en salud. Estos tres principios y las acciones en salud se deben enmarcar con un enfoque intercultural y enfoque de género 30.

La incorporación del enfoque intercultural en la red asistencial de la atención primaria de salud en Chile comenzó en la región de la Araucanía en el año 1996 y se vio respaldada con la reforma sanitaria en el año 2005. Si bien este enfoque apuntaba inicialmente a los pueblos originarios, en la actualidad, el Ministerio de Salud de Chile (MINSAL) 31 (p. 19) señala que en "el enfoque intercultural se deberían incorporar estrategias de pertinencia cultural, que respondan a las particularidades de los distintos pueblos originarios y de la población extranjera que vive en el país, al igual que en las políticas de atención, prevención, rehabilitación y promoción de la salud, con el fin de que sean diseñadas y evaluadas participativamente". En esta misma línea, establece que los equipos de salud deben otorgar prestaciones sanitarias respetando la diversidad cultural de las comunidades que atiende. Entre las recomendaciones que establece el MINSAL para la incorporación del enfoque intercultural al modelo integral de salud familiar y comunitaria se encuentran: programas de formación y capacitación continua en interculturalidad en salud; programas de inducción en salud intercultural a los nuevos funcionarios, especialmente en aquellos sectores que atienden a pueblos originarios; y dotación a los equipos de salud de herramientas de participación que incorporen el conocimiento de la cultura de los usuarios y comunidad.

Acorde a lo anterior, se hace necesario reflexionar de manera crítica sobre este fenómeno y preguntar si los funcionarios sanitarios se encuentran preparados para incorporar el enfoque intercultural en las prestaciones sanitarias, y para establecer una atención y cuidados culturalmente sensibles 32 .

\section{Desarrollo de competencias interculturales en salud para la atención primaria desde la formación inicial y continua en la educación superior}

Dotar a la atención primaria en salud con recursos humanos en número suficiente y adecuadamente capacitados para otorgar una atención en salud integradora con enfoque intercultural, es uno de los retos claves para fortalecer este nivel de atención 33 . La renovación en la atención primaria de salud exige una transformación profunda en los programas de formación de los profesionales de la salud. Al respecto, uno de los elementos definitorios en la educación de pregrado, y permanente en los equipos de salud, es su orientación hacia y desde el trabajo cotidiano, la programación del aprendizaje desde la realidad laboral y social, y la orientación hacia mejoras de desempeño utilizando el enfoque de competencias 33. Las competencias deben ser consideradas como parte de la capacidad adaptativa cognitivo-conductual que es inherente al ser humano, las cuales son desplegadas para responder a las necesidades específicas que las personas enfrentan en contextos sociohistóricos y culturales concretos, lo que implica un proceso de adecuación entre la persona, la demanda del medio y las necesidades que se producen, con la finalidad de poder dar soluciones a las demandas planteadas 34. Por lo tanto, las competencias a desarrollar contribuirían a dominar los instrumentos socioculturales necesarios para interactuar con el conocimiento, permitir la interacción en grupos heterogéneos, potenciar el actuar de un modo autónomo y comprender el contexto. Desde esta mirada, las competencias persiguen una convergencia entre los saberes y el manejo de las interacciones sociales, las emociones y los sentimientos, así como la capacidad de reconocer, interpretar y aceptar a los demás 34.

Para el desarrollo de las competencias interculturales, en los equipos de salud y profesionales en formación, es necesario una mirada transdisciplinaria, que al mismo tiempo nutra y fortalezca el pensamiento complejo, crítico y creativo. La transdisciplinariedad no busca el dominio de muchas disciplinas, sino la apertura de todas aquellas que trasciendan y atraviesen hacia el abordaje intercultural 
de las necesidades en salud. Esta perspectiva pretende unir, articular y vincular saberes, dado que los problemas sanitarios nunca están aislados, sino que son la manifestación de la dinámica social en conjunto con los determinantes de la salud 34 .

La incorporación de conocimientos desde la psicología, antropología, sociología, filosofía, entre otros, en los currículos de formación de los profesionales de la salud, tanto de pregrado como formación continua, es esencial para el desarrollo de competencias interculturales. En general, actualmente, esto se visualiza en los currículos como programas parcelados que no interaccionan entre sí. Es necesario que los saberes se fusionen tanto en el aula, como en las experiencias clínicas o prácticas, para generar la oportunidad en los equipos de salud y estudiantes de pregrado, la capacidad de articular e integrar tanto los conocimientos formales entregados en la institución académica, como los conocimientos personales que van de la mano con las experiencias de vida. Esto, con el fin de abordar desde un modelo pertinente la diversidad cultural a la que el equipo de salud se encuentra expuesto en una sociedad dinámica, globalizada, en donde se entrelazan las migraciones y el contacto interétnico.

El desarrollo de competencias interculturales, tanto a nivel de pregrado como en formación continua, debería incorporar distintos elementos que van más allá de un currículo o programas parcelados, más bien implica una filosofía de vida o una forma de visualizar a la sociedad y la humanidad como un entrelazado diverso y dinámico.

Cuando los profesionales de la salud y estudiantes de pregrado han sido capaces de involucrar la integración de conciencia cultural, conocimientos, encuentros y deseos culturales, se dice que lograron la competencia cultural 35 .

Diversos autores 14,36 han señalado que el problema de las diferencias culturales en el sistema sanitario reside en gran parte en el proceso de comunicación entre el equipo de salud y usuarios. En esta misma línea, señala que la competencia cultural puede garantizar que todo usuario del sistema de salud reciba un tratamiento efectivo e igualitario de una manera cultural y lingüísticamente apropiada.

Según Marrero-González 37, la adquisición de competencias culturales por parte del equipo de salud aborda elementos tales como: (i) el deseo cultural, que es el interés para conocer otras culturas, (ii) el conocimiento cultural, que se relaciona con el aprendizaje sobre la otra cultura, (iii) la conciencia cultural, que se establece una vez que ya se ha conocido la otra cultura, (iv) los encuentros culturales, dadas por las interacciones de personas de distintas culturas, y (v) las habilidades culturales, que son el conjunto de capacidades de respuestas a las necesidades en salud de las personas de otra cultura. También implica el aprendizaje de habilidades como la empatía, la sensibilidad cultural y la reflexión acerca de sus propios valores culturales como un ejercicio necesario para traspasar estas barreras 14 .

Acorde con lo anterior, podríamos establecer que el deseo y el conocimiento cultural podrían ser los cimientos para desarrollar y fortalecer la atención y cuidados en salud con un enfoque intercultural. De acuerdo a Mareno \& Hart 24, existen diversas estrategias pedagógicas que pueden ser implementadas en el aula en educación superior, a partir del diseño de mallas curriculares en el área de la salud. Por ejemplo, se podrían realizar ejercicios orientados a tomar consciencia de la cultura de los mismos estudiantes en los cursos troncales. Además, desarrollar estas competencias interculturales implicaría el diseño de cursos en áreas como teoría y conceptos de las competencias interculturales, que incluyesen aspectos tales como: comunicación entre culturas diversas, alfabetización en salud, inequidad en salud, y promoción en salud. Estos aspectos, según Manero \& Hart 23, deben ser enseñados al inicio de los programas de pregrado y desarrollados transversalmente a lo largo del currículum. Otras estrategias son: análisis de estudios de casos clínicos y juego de roles, aplicados, por ejemplo, en programas de enfermería; simulaciones en una variedad de escenarios para que estudiantes de pre y postgrado puedan practicar la comunicación intercultural con pacientes y familias con una capacidad limitada de expresarse en idioma español 38; brindar a los estudiantes de voluntariados en poblaciones en situación de vulnerabilidad puede aumentar la autoconfianza, cada vez que se encuentren en situaciones de atención en salud a personas pertenecientes a diversas culturas. Sumado a lo anterior, se proponen otras estrategias relacionadas con la inclusión de estudiantes pertenecientes a pueblos originarios o a países con población migrante en los programas de pregrado del área de la salud 39, como el uso de películas; a través de ellas, los docentes pueden exponer a los estudiantes a una variedad de nuevas situaciones y grupos culturales en un corto periodo de tiempo 38 . 
Un currículo que incorpore, además, el conocimiento en etnobotánica, saberes tradicionales de diversas culturas, enseñados por una autoridad indígena en dichos saberes, e incluso el aprendizaje de los idiomas usados por las diversas etnias con las cuales los estudiantes atenderán son parte de la variedad de estrategias que pueden implementarse en educación superior para el aprendizaje y la práctica de competencias interculturales 40.

En situaciones de práctica profesional es necesario que los profesores establezcan espacios de reflexión sobre dichas experiencias para fomentar la reflexión individual y colectiva sobre qué resultó bien y por qué y cómo podrían mejorar en futuras situaciones 24. En cuanto a las experiencias clínicas y trabajo en terreno de atención primaria de salud, es posible incluir actividades destinadas a que los estudiantes fortalezcan sus competencias interculturales en el contexto del modelo integral de salud familiar. En este escenario, surgen como opciones interesantes el desarrollo de diagnósticos comunitarios participativos, como también la formulación de proyectos comunitarios en salud rural; la creación e implementación de actividades de promoción y prevención con participación activa de la población; y la incorporación del trabajo interdisciplinario en el desarrollo de las experiencias clínicas. Finalmente, es posible implementar pasantías en programas de salud intercultural, a cargo tanto del Estado como de organizaciones no gubernamentales relacionadas, y desarrollar actividades de aprendizaje - servicio que suponen una relación directa con las experiencias cotidianas de comunidades socioculturalmente diversas.

\section{Conclusión}

El escenario de la globalización, las continuas migraciones desde y hacia diferentes lugares del orbe, la importancia de las minorías étnicas y el respeto a la diversidad cultural constituyen hoy en día un desafío para los profesionales y equipos de salud en la atención primaria, en tanto suponen un abordaje distinto, tanto de los problemas de salud, como de la interacción con los usuarios en relación a lo que históricamente impuso el modelo biomédico. El desarrollo de competencias interculturales en los estudiantes del área de la salud permitiría a los futuros profesionales de la atención primaria desempeñar su rol con adecuada pertinencia cultural, asumiendo las características que impone la época actual tanto a la relación con los usuarios, como a su comprensión, en tanto seres holísticos y culturalmente diversos. La adquisición de tales competencias es imperiosa en el contexto socio-histórico presente, con el fin de considerar la diversidad cultural como un elemento que favorece las intervenciones de los equipos de salud en el nivel de la atención primaria. Es urgente fortalecer la formación que reciben los estudiantes del área de la salud hoy en día en este ámbito, incorporando elementos que faciliten su comprensión del otro culturalmente distinto, y los modos en que se actúa en términos profesionales en un contexto de diversidad cultural. Lo anterior permitirá la consolidación de una cosmovisión holística del ser, por parte de los estudiantes en su periodo formativo y un adecuado desempeño en escenarios interculturales en su ejercicio profesional futuro. 


\section{Colaboradores}

L. Veliz-Rojas contribuyó con la elaboración de tesis central, elaboración de relaciones entre educación y salud, desarrollo conceptual en salud, revisión bibliográfica y edición final del texto. A. F. Bianchetti-Saavedra contribuyó con la revisión bibliográfica, identificación y elaboración de propuestas para el desarrollo de competencias interculturales, elaboración de relaciones entre terminología antropológica y educación, y edición final del texto. M. Silva-Fernández contribuyó con la revisión bibliográfica, desarrollo conceptual de la terminología antropológica y edición final del texto.

\section{Referencias}

1. Duqué-Páramo M. Cultura y salud: elementos para el estudio de la diversidad y las inequidades. Investigación en Enfermería: Imagen y Desarrollo 2007; 9:123-38.

2. Organización Mundial de la Salud; Fondo de las Naciones Unidas para la Infancia. Declaración de Alma-Ata. Geneva: Organización Mundial de la Salud/Fondo de las Naciones Unidas para la Infancia; 1988.

3. Mundel E, Chapman GE. A decolonizing approach to health promotion in Canada: the case of the Urban Aboriginal Community Kitchen Garden Project. Health Promot Int 2010; 25:166-73.

4. Langdon EJ, Wiik FB. Antropología, salud y enfermedad: una introducción al concepto de cultura aplicado a las ciencias de la salud. Rev Latinoam Enferm 2010; 18:459-66.

5. Schwandt TA. The SAGE dictionary of qualitative inquiry. Thousand Oaks: SAGE Publications; 2015.

6. Geertz C. La interpretación de las culturas. Barcelona: Editorial GEDISA; 1973.

7. Leininger M, McFarland M. Transcultural nursing: concepts, theories, research \& practice. 3rd Ed. New York: McGraw-Hill; 2002.

8. Guilherme M, Dietz G. Diferencia en la diversidad: perspectivas múltiples de complejidades conceptuales multi, inter y transculturales. Estudios sobre las Culturas Contemporáneas 2014; 20:13-36.

9. Deardorff DK. The SAGE handbook of intercultural competence. Thousand Oaks: SAGE Publications; 2009.

10. Murillo J. Construcción de las competencias interculturales para el desarrollo de una propuesta de experiencias de aprendizaje para alumnos de primer año de medicina. An Fac Med (Perú) 2015; 76:77-87.

11. Chaib M. Interculturalidad y formación en ciencias de la salud. Educ Méd 2005; 8:42-50.

12. Rojas N, Amode N, Vásquez J. Racismo y matrices de "inclusión" de la migración haitiana en Chile: elementos conceptuales y contextuales para la discusión. Polis: Revista Latinoamericana 2015 ; $14: 217-45$.

13. Bernales M, Cabieses B, McIntyre A, Chepo M. Desafíos en la atención sanitaria de migrantes internacionales en Chile. Rev Peru Med Exp Salud Pública 2017; 34:167-75

14. Scozia C, Leiva C, Garrido N, Álvarez A. Barreras interaccionales en la atención maternoinfantil a inmigrantes peruanas. Revista Sociedad y Equidad 2014; 6:6-33.

15. Cabieses B, Chepo M, Oyarte M, Markkula N, Bustos P, Pedrero V, et al. Brechas de desigualdad en salud en niños migrantes versus locales en Chile. Rev Chil Pediatr 2017; 88:707-16.

16. Hasen F. Interculturalidad en salud: competencias prácticas de salud con población indígena. Cienc Enferm 2012; 18:17-24. 
17. Piñeiro E. Educación médica para la inclusión intercultural desde un estudio de caso entre los Mbyá-guaraní. Educ Méd Super 2015; 29:82431.

18. Alarcón A, Astudillo P, Barrios S, Rivas E. Política de salud intercultural: perspectiva de usuarios mapuches y equipo de salud en la IX región, Chile. Rev Méd Chile 2004; 132:110914.

19. Organización Panamericana de la Salud. Salud de los pueblos indígenas de las Américas. Rev Panam Salud Pública 1998; 3:278-84.

20. Vila D. El concepto del buen vivir/Sumak Kawsay desde la perspectiva de la gubermentalidad. Delimitación, innovaciones y conflictos. Revista de Antropología Experimental 2014; 4:47-60.

21. Arteaga-Cruz K. Buen vivir (Sumak Kawsay): definiciones, crítica e implementaciones en la planificación del desarrollo en Ecuador. Saúde Debate 2017; 41:907-19.

22. Caria S, Domínguez R. Ecuador's Buen vivir: a new ideology for development. Latin American Perspectives 2016; 43:18-33.

23. Menéndez E. Salud intercultural: propuestas, acciones y fracasos. Ciênc Saúde Coletiva 2016; 21:109-18.

24. Mareno N, Hart PL. Cultural competency among nurses with undergraduate and graduate degrees: implications for nursing education. Nurs Educ Perspect 2014; 35:83-8.

25. Organización de las Naciones Unidas para la Educación, la Ciencia y la Cultura. Declaración universal sobre la diversidad cultural. Paris: Organización de las Naciones Unidas para la Educación, la Ciencia y la Cultura; 2004. (Serie sobre la Diversidad Cultural, 1).

26. Osorio-Merchán M, López A. Competencia cultural en salud: necesidad emergente en un mundo globalizado. Index Enferm 2008; 17:266-70.

27. Janer M. Diversidad y competencias profesionales interculturales. Educación Social 2013; 54:39-48.

28. Kleimann A, Benson P. Anthropology in the clinic. The problemof cultural competency and how to fix. PLoS Med 2006; 3:e294.

29. Garita A. La orientación intercultural: una perspectiva para favorecer la convivencia en contextos multiculturales. Educare 2014; 18:281-91.

30. Dois A, Contreras A, Bravo P, Mora I, Soto G, Solís C. Principios orientadores del modelo de salud familiar y comunitario desde la perspectiva de los usuarios. Rev Méd Chile 2016; 144:585-92.
31. División de Atención Primaria, Subsecretaría de Redes Asistenciales, Ministerio de Salud de Chile. Orientaciones para la implementación del modelo de atención integral de salud familiar y comunitaria. Dirigido a equipos de salud. https://www.minsal.cl/portal/url/item/ e7b24eef3e5cb5d1e0400101650128e9.pdf (accedido el 20/Abr/2018)

32. Jofré S, Sepúlveda C. Migración en Chile: un nuevo desafío para la formación de competencias culturales en estudiantes de enfermería. Enferm Actual Costa Rica (Online) 2017; (33). https://revistas.ucr.ac.cr/index.php/enferme ria/article/view/28950.

33. Nebot C, Rosales C, Borell R. Desarrollo de competencias en atención primaria de salud. Rev Panam Salud Pública 2009; 26:176-83.

34. García J. Modelo educativo basado en competencias: importancia y necesidad. Actualidades Investigativas en Educación 2011; 11:1-24.

35. Escobar B, Paravic T. La transculturalidad, elemento esencial para mejorar la atención en salud y de enfermería. Enferm Actual Costa Rica (Online) 2017; (32). https://revistas.ucr.ac.cr/ index.php/enfermeria/article/view/29627.

36. Gourlat BG, Levey S, Soares RS. Multiculturality skills, health care and communication disorders. Cad Saúde Pública 2018; 34:e00217217.

37. Marrero-González CM. Competencia cultural. Enfoque del modelo de Purnell y Campinha-Bacote en la práctica de profesiones sanitarias. Ene 2013; 7(2). http://www.eneenfermeria.org/ojs/index.php/ENE/article/ viewFile/278/pdf_3.

38. Jeffreys MR. Teaching cultural competence in nursing and health care: inquiry, action, and innovation. New York: Springer Publishing Company; 2015.

39. Betancourt JR, Green AR, Carrillo JE, AnanehFirempong O. Defining cultural competence: a practical framework for addressing racial/ethnic disparities in health and health care. Public Health Rep 2016; 118:293-302.

40. Gallegos-Zurita M. Las plantas medicinales: principal alternativa para el cuidado de la salud, en la población rural de Babahoyo, Ecuador. An Fac Med (Perú) 2016; 77:327-32. 


\section{Abstract}

Contemporary society is marked by four interconnected phenomena leading to the need to develop intercultural skills in the framework of primary care: migratory movements, different ethnic groups, cultural diversity, and social and cultural construction of the Western medical system. The multicultural reality of the contexts in which primary care is practiced requires integrative theoretical models that allow understanding this reality in order to provide timely, high-quality care that is pertinent to the respective communities, considering the existing diversity. It is thus necessary to implement and teach intercultural skills for health care teams and students, allowing to sustain such care from a holistic perspective. Educational interventions in intercultural skills should be conducted from a comprehensive, integrative, and cross-disciplinary perspective that allows intervening in attitudes, feelings, social behaviors, and cultural agendas at the individual, institutional, and social levels. The current essay aims to reflect on the importance of learning and developing such skills in primary care teams, meanwhile producing a list of strategies that allow acquiring these skills in basic training, with the understanding that skills are the result of experiences and can change during the process.

Cultural Competency; Cultural Diversity; Primary Health Care; Higher Education; Health Occupations Students

\section{Resumo}

$\mathrm{Na}$ sociedade atual estão presentes quatro fenômenos articulados que determinam a necessidade de desenvolver competências interculturais no âmbito da atenção primaria à saúde: os movimentos migratórios, as diferentes etnias, a diversidade cultural e a construção social e cultural do sistema médico ocidental. A realidade multicultural dos contextos nos quais é executada a atenção sanitária exige incorporar modelos teóricos integradores que permitam entendê-la para poder oferecer uma atenção oportuna na saúde, para que seja de qualidade e pertinente para as comunidades a quem estão focadas suas ações, levando em consideração a diversidade existente. Neste sentido, é necessária a implantação e o ensino de competências interculturais nas equipes e estudantes da área de saúde, que permitam sustentar a atenção e os cuidados desde uma perspectiva holistica. A intervenção educativa em competência intercultural deve ser realizada desde um enfoque global, integrador e transdisciplinar, que permita intervir desde as atitudes, os sentimentos, as condutas sociais e as pautas culturais ao nível individual, institucional e social. O presente ensaio tem como propósito reflexionar sobre a importância da aprendizagem e desenvolvimento de ditas competências nas equipes de saúde de atenção primaria e enumerar uma listagem de estratégias com as quais é possível adquiri-las nos processos formativos prévios, entendendo que as competências são fruto das experiências e podem ser transformadas em resultado de um processo.

Competência Cultural; Diversidade Cultural; Atenção Primária à Saúde; Educação Superior; Estudantes de Ciências da Saúde
Recibido el 18/Jun/2018

Versión final presentada el 26/Ago/2018

Aprobado el 24/Sep/2018 